\title{
A transference inequality for rational approximation to points in geometric progression
}

\author{
Jérémy Champagne and Damien Roy
}

Dedicated to the memory of Alan Baker

\begin{abstract}
We establish a transference inequality conjectured by Badziahin and Bugeaud relating exponents of rational approximation of points in geometric progression.

Keywords. exponents of Diophantine approximation, Hausdorff dimension, polynomials, transference inequalities.

2010 Mathematics Subject Classification. Primary 11J13; Secondary 11J82, 11J83.
\end{abstract}

Let $\xi \in \mathbb{R}$. For each integer $n \geq 1$, one defines $\omega_{n}(\xi)$ as the supremum of all $\omega \in \mathbb{R}$ for which there exist infinitely many non-zero polynomials $P(x) \in \mathbb{Z}[x]$ of degree at most $n$ with

$$
|P(\xi)| \leq\|P\|^{-\omega}
$$

where $\|P\|$ stands for the largest absolute value of the coefficients of $P$. These quantities form the basis of Mahler's classification of numbers [Mah32]. Dually, one defines $\lambda_{n}(\xi)$ as the supremum of all $\lambda \in \mathbb{R}$ for which there exist infinitely many non-zero points $\mathbf{x}=\left(x_{0}, \ldots, x_{n}\right) \in \mathbb{Z}^{n+1}$ such that

$$
\max _{1 \leq m \leq n}\left|x_{0} \xi^{m}-x_{m}\right| \leq\|\mathbf{x}\|^{-\lambda}
$$

where $\|\mathbf{x}\|$ stands for the maximum norm of $\mathbf{x}$.

Suppose now that $\xi$ is transcendental over $\mathbb{Q}$, i.e. that $\xi \in \mathbb{R} \backslash \overline{\mathbb{Q}}$. In [BaBu19], D. Badziahin and Y. Bugeaud prove that for any integers $k, n$ with $2 \leq k \leq n$, we have

$$
\lambda_{n}(\xi) \geq \frac{\omega_{k}^{\text {lead }}(\xi)-n+k}{(k-1) \omega_{k}^{\text {lead }}(\xi)+n},
$$

where $\omega_{k}^{\text {lead }}(\xi)$ is defined as $\omega_{k}(\xi)$ but by restricting to polynomials $P(x) \in \mathbb{Z}[x]$ of degree $k$ whose coefficient $c_{k}(P)$ of $x^{k}$ has largest absolute value $\left|c_{k}(P)\right|=\|P\|$. They conjecture that this inequality remains true if $\omega_{k}^{\text {lead }}(\xi)$ is replaced by $\omega_{k}(\xi)$ and they prove this is indeed the case if $k=2$ or $k=n-1$. Their proof for $k=2$ is based on the fact that $\lambda_{n}(\xi)=\lambda_{n}(1 / \xi)$. The purpose of this note is to prove this conjecture based on their inequality (1), the invariance of $\lambda_{n}$ and $\omega_{k}$ by general fractional linear transformations with rational coefficients, and the following observation.

Theorem. Let $k \geq 1$ be an integer and let $r_{0}, \ldots, r_{k}$ be distinct integers. There is an integer $M \geq 1$ such that, for each $\xi \in \mathbb{R}$, there exists at least one index $i \in\{0,1, \ldots, k\}$, with $r_{i} \neq \xi$, for which the point $\xi_{i}=1 /\left(M\left(\xi-r_{i}\right)\right)$ satisfies $\omega_{k}^{\text {lead }}\left(\xi_{i}\right)=\omega_{k}\left(\xi_{i}\right)=\omega_{k}(\xi)$.

Proof. We first note that there exist positive constants $C_{1}$ and $C_{2}$ such that

$$
\|P\| \leq C_{1} \max \left\{\left|P\left(r_{i}\right)\right| ; 0 \leq i \leq k\right\} \quad \text { and } \max \left\{\left\|P\left(x+r_{i}\right)\right\| ; 0 \leq i \leq k\right\} \leq C_{2}\|P\|
$$

for any polynomial $P \in \mathbb{R}[x]$ of degree at most $k$. Let $\xi \in \mathbb{R}$. Choose an integer $M$ with $M \geq C_{1} C_{2}$ and a sequence of polynomials $\left(P_{j}\right)_{j \geq 1}$ in $\mathbb{Z}[x]$ of degree at most $k$ with strictly increasing norms such

Work of the authors partially supported by NSERC

We thank episciences.org for providing open access hosting of the electronic journal Hardy-Ramanujan Journal 
that

$$
\lim _{j \rightarrow \infty}-\frac{\log \left|P_{j}(\xi)\right|}{\log \left\|P_{j}\right\|}=\omega_{k}(\xi)
$$

Then, choose $i \in\{0,1, \ldots, k\}$ such that $\left\|P_{j}\right\| \leq C_{1}\left|P_{j}\left(r_{i}\right)\right|$ for an infinite set $S$ of positive integers $j$, and set

$$
Q_{j}(x)=(M x)^{k} P_{j}\left(\frac{1}{M x}+r_{i}\right) \in \mathbb{Z}[x]
$$

for each $j \in S$. For those values of $j$, the absolute value of the coefficient of $x^{k}$ in $Q_{j}(x)$ is $\left|c_{k}\left(Q_{j}\right)\right|=$ $M^{k}\left|P_{j}\left(r_{i}\right)\right|$ while its other coefficients have absolute value at most

$$
M^{k-1}\left\|P_{j}\left(x+r_{i}\right)\right\| \leq C_{2} M^{k-1}\left\|P_{j}\right\| \leq C_{1} C_{2} M^{k-1}\left|P_{j}\left(r_{i}\right)\right| \leq M^{k}\left|P_{j}\left(r_{i}\right)\right|,
$$

thus $\left|c_{k}\left(Q_{j}\right)\right|=\left\|Q_{j}\right\|$. We also have $r_{i} \neq \xi$ and

$$
\left|Q_{j}\left(\xi_{i}\right)\right|=\left(M\left|\xi_{i}\right|\right)^{k}\left|P_{j}(\xi)\right|
$$

where $\xi_{i}=1 /\left(M\left(\xi-r_{i}\right)\right)$. As the ratio $\left\|Q_{j}\right\| /\left\|P_{j}\right\|$ is bounded from above and from below by positive constants, we deduce from (2) that $-\log \left|Q_{j}\left(\xi_{i}\right)\right| / \log \left\|Q_{j}\right\|$ converges to $\omega_{k}(\xi)$ as $j$ goes to infinity in $S$. Altogether, this means that $\omega_{k}^{\text {lead }}\left(\xi_{i}\right) \geq \omega_{k}(\xi)$. However, it is well known (and easy to prove) that $\omega_{k}(\xi)=\omega_{k}\left(\xi_{i}\right)$ because $\xi_{i}$ is the image of $\xi$ by a linear fractional transformation with rational coefficients. The conclusion follows because $\omega_{k}\left(\xi_{i}\right) \geq \omega_{k}^{\text {lead }}\left(\xi_{i}\right)$ by the very definition of $\omega_{k}^{\text {lead }}$.

Applying the formula (1) with $\xi$ replaced by $\xi_{i}$ and using the fact that $\lambda_{n}\left(\xi_{i}\right)=\lambda_{n}(\xi)$, we reach the desired inequality.

Corollary 1. $\lambda_{n}(\xi) \geq \frac{\omega_{k}(\xi)-n+k}{(k-1) \omega_{k}(\xi)+n}$ for any $\xi \in \mathbb{R} \backslash \overline{\mathbb{Q}}$ and any integers $2 \leq k \leq n$.

We thank Yann Bugeaud and Victor Beresnevich for pointing out that our theorem formalizes principles that are implicit in Lemmas 3 and 4 of [Bak76] as well as on pages 25-26 of [Spr69] (for the choice of $r_{i}=i$ ). The next corollary is an application to metrical theory that was suggested by Yann Bugeaud.

Corollary 2. Let $k \geq 1$ be an integer and let $w \in \mathbb{R}$. The sets $S=\left\{\xi \in \mathbb{R} ; \omega_{k}(\xi) \geq w\right\}$ and $S^{\text {lead }}=\left\{\xi \in \mathbb{R} ; \omega_{k}^{\text {lead }}(\xi) \geq w\right\}$ have the same Hausdorff dimension. This remains true if we replace the large inequalities $\geq$ by strict inequalities $>$ in the definitions of $S$ and $S^{\text {lead }}$.

Proof. We have $S^{\text {lead }} \subset S$ and, for an appropriate choice of integers $r_{0}, \ldots, r_{k}$ and $M$, the theorem gives $S \subseteq \bigcup_{i=0}^{k} \tau_{i}^{-1}\left(S^{\text {lead }}\right)$ where $\tau_{i}(\xi)=1 /\left(M\left(\xi-r_{i}\right)\right)$ for each $\xi \in \mathbb{R} \backslash\left\{r_{i}\right\}(0 \leq i \leq k)$. The conclusion follows by the invariance of the Hausdorff dimension under fractional linear transformations.

\section{References}

[BaBu19] D. Badziahin and Y. Bugeaud, On simultaneous rational approximation to a real number and its integral powers II, New York J. Math. 26 (2020), 362-377; e-print 13 June 2019, arXiv:1906.05508, 17 pages.

[Bak76] R. C. Baker, Sprindžuk's theorem and Hausdorff dimension, Mathematika 23 (1976), $184-197$.

[Mah32] K. Mahler, Zur Approximation der Exponentialfunktion und des Logarithmus. Teil I, J. Reine Angew. Math. 166 (1932), 118-136.

[Spr69] V. G. Sprindžuk, Mahler's problem in metric number theory, Translations of Mathematical Monographs, Vol. 25, American Mathematical Society, Providence, R.I. 1969, vii+192 pp. 


\section{Jérémy Champagne}

Département de Mathématiques

Université d'Ottawa

150 Louis Pasteur

Ottawa, Ontario K1N 6N5, Canada

e-mail: jcham016@uottawa.ca

\section{Damien Roy}

Département de Mathématiques

Université d'Ottawa

150 Louis Pasteur

Ottawa, Ontario K1N 6N5, Canada

e-mail: droy@uottawa.ca 